PROCEEDINGS OF THE

AMERICAN MATHEMATICAL SOCIETY

Volume 139, Number 10, October 2011, Pages 3475-3483

S 0002-9939(2011)11124-9

Article electronically published on June 6, 2011

\title{
GLOBAL ASYMPTOTIC STABILITY FOR PREDATOR-PREY SYSTEMS WHOSE PREY RECEIVES TIME-VARIATION OF THE ENVIRONMENT
}

\author{
JITSURO SUGIE, YASUHISA SAITO, AND MENG FAN
}

(Communicated by Yingfei Yi)

\begin{abstract}
A predator-prey model with prey receiving time-variation of the environment is considered. Such a system is shown to have a unique interior equilibrium that is globally asymptotically stable if the time-variation is bounded and weakly integrally positive. In particular, the result tells us that the equilibrium point can be stabilized even by nonnegative functions that make the limiting system structurally unstable. The method that is used to obtain the result is an analysis of asymptotic behavior of the solutions of an equivalent system to the predator-prey model.
\end{abstract}

\section{INTRODUCTION}

Predator-prey systems in nature apparently persist stably while the most basic models and experiments show their instability (see, for example, 8, 17, 23, 31]). This gap suggests that our insight is not enough to understand mechanisms acting in nature which stabilize population dynamics. To resolve the gap, theoreticians and experimentalists have made a long list of such processes (see the books [6, 14, [18, 21]).

The basic theoretical tool in these investigations is the system of Lotka-Volterra equations for a prey with population density $N(t)$ and a predator with population density $P(t)$ :

$$
\begin{aligned}
& N^{\prime}=(a-b P) N, \\
& P^{\prime}=(-c+d N) P,
\end{aligned}
$$

where the prime denotes $d / d t$ and the parameters $a, b, c$, and $d$ are assumed to be positive. This model is often criticized because its single positive equilibrium point is a center, i.e., a "neutrally stable" equilibrium surrounded by a family of periodic orbits whose amplitudes depend on the initial population sizes. Also, the slightest change to the model's structure typically results in qualitatively different behavior. For example, if $a$ decreases linearly with prey density the equilibrium point is stable.

Received by the editors June 12, 2010.

2010 Mathematics Subject Classification. Primary 34D23, 92D25; Secondary 34D05, 37B25.

Key words and phrases. Global asymptotic stability, predator-prey systems, weakly integrally positive, time-variation.

The first author was supported in part by Grant-in-Aid for Scientific Research No. 22540190 from the Japan Society for the Promotion of Science.

The third author was supported in part by the NSFC, NCET-08-0755 and FRFCU. 
On the other hand, introducing a saturating (Type II) functional response turns the equilibrium into an unstable spiral point (see [9]). This structural instability means that the model cannot make any predictions that are robust enough to be tested. After all, we know that model $(\underline{L V})$ does not adequately describe even the most highly controlled experiments.

Structural instability can, however, be used to our advantage. In effect, it allows us to use the Lotka-Volterra equations as an exquisitely sensitive balance, with which we can determine the effects of the processes that it ignores. So, when we say that a Type II functional response is destabilizing, we mean that it destabilizes the equilibrium point in model $(L V)$. Similarly, when we say that the presence of a carrying capacity for the prey tends to be stabilizing, we mean that it stabilizes the equilibrium point. There is a long tradition of using the Lotka-Volterra equations in this way (see 22]).

A time-variational component of the environment is one of the processes that the Lotka-Volterra equations ignore. Realistic models should take account of seasonal effect. Constant per capita birth and mortality rates are highly unlikely for most natural populations; rather they are usually subject to seasonal fluctuations. Supposing that prey have a carrying capacity and are more effective to receive timevariation of the environment than their predators, we may discuss a general version of model $(L V]$ where only $a$ is modified to $a=\beta(t)-\delta(t) N$ with continuous and nonnegative functions $\beta(t)$ and $\delta(t)$.

In this paper, in a simple way, we consider a predator-prey model of the form

$$
\begin{aligned}
N^{\prime} & =(a+c h(t)-d h(t) N-b P) N, \\
P^{\prime} & =(-c+d N) P,
\end{aligned}
$$

where $h(t)$ is continuous and nonnegative for $t \geq 0$ and $a, b, c$, and $d$ are positive constants. It is clear that the modified model (E) still has a unique interior equilibrium point $(c / d, a / b)$. Needless to say, we only have to consider model $(E)$ in the first quadrant $\{(N, P): N>0$ and $P>0\}$. Hence, the initial data is in the first quadrant. The interior equilibrium of $(E)$ is said to be globally attractive if it attracts any solution of $(E)$ with the initial data. Moreover, if interior equilibrium of $(E)$ is stable, then it is said to be globally asymptotically stable.

To state our main result, we define a family of functions. We say that a nonnegative function $\phi$ is weakly integrally positive if

$$
\int_{I} \phi(t) d t=\infty
$$

for every set $I=\bigcup_{n=1}^{\infty}\left[\tau_{n}, \sigma_{n}\right]$ such that $\tau_{n}+\delta<\sigma_{n}<\tau_{n+1} \leq \sigma_{n}+\Delta$ for some $\delta>0$ and $\Delta>0$. A simple example of a weakly integrally positive function is $\sin ^{2} t, 1 /(1+t)$, or $\sin ^{2} t /(1+t)$ (see [12, 13, 27, 28, 29]). It is easy to see that the family of weakly integrally positive functions includes nonnegative functions which converge to 0 as $t \rightarrow \infty$.

Theorem 1. Suppose there exists an $\bar{h}$ such that $0 \leq h(t) \leq \bar{h}$ for $t \geq 0$. If $h(t)$ is weakly integrally positive, then the interior equilibrium $(c / d, a / b)$ of $(E)$ is globally asymptotically stable.

Theorem 1 tells us that the equilibrium point of $(E)$ can be stabilized even by such nonnegative functions that make the limiting system of $(E)$ structurally unstable. 
The organization of this paper is as follows. In Section 2, we introduce a transformation and establish a proposition on Lyapunov's stability. Also, we examine properties of certain functions which will be used in proving our main theorem. We prove the main theorem in Section 3 and summarize our findings in Section 4.

\section{TRANSFORMATION}

Changing variables

$$
x=-\log (b P / a) \quad \text { and } \quad y=-\log (d N / c),
$$

we can transform model $(E)$ into the system

$$
\begin{aligned}
& x^{\prime}=c\left(1-e^{-y}\right), \\
& y^{\prime}=-a\left(1-e^{-x}\right)-c h(t)\left(1-e^{-y}\right) .
\end{aligned}
$$

System (10) has the zero solution $(x(t), y(t)) \equiv(0,0)$, which corresponds to the interior equilibrium $(c / d, a / b)$ of $(E)$. The above transformation is a one-to-one correspondence from the first quadrant $\{(N, P): N>0$ and $P>0\}$ to the whole real plane $\{(x, y): x \in \mathbb{R}$ and $y \in \mathbb{R}\}$. Hence, the interior equilibrium $(c / d, a / b)$ of $(E)$ is globally attractive if and only if every solution $(x(t), y(t))$ of (1) tends to $(0,0)$ as time $t$ increases.

To prove Theorem 1, we have to derive two conclusions: the zero solution of (1) is stable; any solution of (11) approaches the origin $(0,0)$. It is easy to show the stability of the zero solution of (1) by using a Lyapunov-type theorem. For details about the direct method of Lyapunov, see the books [1, 4, 10, 11, 16, 19, 20, 26, 33, 34 for example.

Proposition 2. If $h(t)$ is nonnegative for $t \geq 0$, then the zero solution of (11) is uniformly stable.

Proof. As a suitable Lyapunov function, we choose

$$
V(x, y)=a f(x)+c f(y)
$$

where $f(z)=e^{-z}+z-1$ for $z \in \mathbb{R}$. Since

$$
\frac{d}{d z} f(z)=-e^{-z}+1
$$

$f(z)$ is increasing for $z \geq 0$ and decreasing for $z \leq 0$. Hence, $f(z)>0=f(0)$ for $z \neq 0$. This means that $V(x, y)$ is positive definite and decrescent. Differentiate $V(x, y)$ along any solution of (11) to obtain

$$
\dot{V}_{\text {[1] }}(t, x, y)=-c^{2} h(t)\left(1-e^{-y}\right)^{2} \leq 0
$$

on $[0, \infty) \times \mathbb{R}^{2}$. We therefore conclude that the zero solution of (11) is uniformly stable by using a Lyapunov-type theorem due to Persidski 25] (refer also to Theorem 1.7 in [26, p. 14] or to Theorem 8.2 in [33, p. 32]).

In order to prove the attraction of any solution of (11), it is helpful to describe the properties of functions $f(z)$ and

$$
g(z)=\left|1-e^{-z}\right|
$$

for $z \in \mathbb{R}$. It is clear that the inequality $f(-z) \geq f(z)$ holds for $z \geq 0$, with equality if and only if $z=0$. Hence, we see that

$$
0 \leq f(z) \leq f(-\alpha) \text { for }|z| \leq \alpha
$$


with $\alpha$ positive. Let $f^{-1}(w)$ be the inverse function of $w=f(z) \operatorname{sgn} z$. Then, $f^{-1}(w)$ is increasing for $w \in \mathbb{R}$ and $f^{-1}(0)=0$. Hence, $f^{-1}(w)$ is positive for $w>0$ and negative for $w<0$. Since $f(-z)>f(z)>0$ for $z>0$, we see that

$$
0<-f^{-1}(-w)<f^{-1}(w) \text { for } w>0 .
$$

Also, $f^{-1}(w)$ tends to $\infty$ as $w \rightarrow \infty$ and it tends to $-\infty$ as $w \rightarrow-\infty$. Since

$$
\frac{d}{d z} g(z)= \begin{cases}e^{-z} & \text { if } z \geq 0 \\ -e^{-z} & \text { if } z \leq 0\end{cases}
$$

$g(z)$ is increasing for $z \geq 0$ and decreasing for $z \leq 0$. Hence, $g(z)>0=g(0)$ for $z \neq 0$. It is easy to check that $\lim _{z \rightarrow \infty} g(z)=1, \lim _{z \rightarrow-\infty} g(z)=\infty$ and the inequality $g(-z) \geq g(z)$ holds for $z \geq 0$, with equality if and only if $z=0$. Hence, we see that

$$
g(z) \geq g(\alpha)>0 \text { for }|z| \geq \alpha
$$

and

$$
0 \leq g(z) \leq g(-\alpha) \text { for }|z| \leq \alpha
$$

with $\alpha$ positive.

\section{Proof of the main Result}

We are now ready to prove Theorem 1.

Proof of Theorem 1. By means of Proposition 2, we conclude that the zero solution of (11) is stable. We will prove that every solution of (1) tends to the origin.

Let $(x(t), y(t))$ be any solution of (1) with the initial time $t_{0} \geq 0$ and let

$$
v(t)=V(x(t), y(t))=a f(x(t))+c f(y(t)) .
$$

Then, we have

$$
v^{\prime}(t)=-c^{2} h(t) g^{2}(y(t)) \leq 0 \text { for } t \geq t_{0} ;
$$

namely, $v(t)$ is nonincreasing for $t \geq t_{0}$. Hence, $v(t)$ has a limiting value $v_{0} \geq 0$. If $v_{0}=0$, then from (66) we see that the solution $(x(t), y(t))$ tends to $(0,0)$ as $t \rightarrow \infty$. This completes the proof. Thus, we need consider only the case in which $v_{0}>0$. We will show that this case does not occur.

Since $v(t)$ tends to a positive value $v_{0}$ as $t \rightarrow \infty$, there exists a $T_{1} \geq t_{0}$ such that

$$
0<v_{0} \leq v(t) \leq 2 v_{0} \text { for } t \geq T_{1}
$$

Hence, by (6), we have

namely,

$$
f(y(t)) \leq \frac{2 v_{0}}{c}
$$

$$
f^{-1}\left(-2 v_{0} / c\right) \leq y(t) \leq f^{-1}\left(2 v_{0} / c\right)
$$

for $t \geq T_{1}$. From (3), we see that

$$
|y(t)| \leq f^{-1}\left(2 v_{0} / c\right) \text { for } t \geq T_{1}
$$

Since $|y(t)|$ is bounded, it has an inferior limit and a superior limit. First, we will show that the inferior limit of $|y(t)|$ is zero, and we will then show that the superior limit of $|y(t)|$ is also zero. 
Suppose that $\liminf _{t \rightarrow \infty}|y(t)|>0$. Then, there exist a $\gamma>0$ and a $T_{2} \geq t_{0}$ such that $|y(t)|>\gamma$ for $t \geq T_{2}$. It follows from (4) that $g^{2}(y(t)) \geq g^{2}(\gamma)$ for $t \geq T_{2}$. Hence, we have

$$
v^{\prime}(t)=-c^{2} h(t) g^{2}(y(t)) \leq-c^{2} g^{2}(\gamma) h(t)
$$

for $t \geq T_{2}$. Integrating this inequality from $t_{0}$ to $t$, we obtain

$$
-v\left(t_{0}\right) \leq v(t)-v\left(t_{0}\right)=\int_{t_{0}}^{t} v^{\prime}(s) d s \leq-c^{2} g^{2}(\gamma) \int_{T_{2}}^{t} h(s) d s,
$$

which tends to $-\infty$ as $t \rightarrow \infty$ because $h(t)$ is weakly integrally positive. This is a contradiction. Thus, we see that $\liminf _{t \rightarrow \infty}|y(t)|=0$.

Suppose that $\limsup _{t \rightarrow \infty}|y(t)|>0$. Let $\lambda=\limsup _{t \rightarrow \infty}|y(t)|$. Let $\varepsilon$ be so small that $0<\varepsilon<-f^{-1}\left(-v_{0} / c\right)$,

$$
\frac{c \bar{h}}{a} g(-\varepsilon)<1-\exp \left(-f^{-1}\left(\frac{v_{0}-c f(-\varepsilon)}{a}\right)\right)
$$

and

$$
\frac{c \bar{h}}{a} g(-\varepsilon)<\exp \left(-f^{-1}\left(\frac{c f(-\varepsilon)-v_{0}}{a}\right)\right)-1
$$

Since $g(-\varepsilon)$ approaches zero and the right-hand sides of (9) and (10) approach positive numbers as $\varepsilon \rightarrow 0$, we can find such a positive number $\varepsilon$. Also, we may assume without loss of generality that $\varepsilon<\lambda / 2$.

As proved above, the inferior limit of $y(t)$ is zero. Hence, we can choose two intervals $\left[\tau_{n}, \sigma_{n}\right]$ and $\left[t_{n}, s_{n}\right]$ with $\left[t_{n}, s_{n}\right] \subset\left[\tau_{n}, \sigma_{n}\right], T_{1}<\tau_{n}$ and $\tau_{n} \rightarrow \infty$ as $n \rightarrow \infty$ such that $\left|y\left(\tau_{n}\right)\right|=\left|y\left(\sigma_{n}\right)\right|=\varepsilon,\left|y\left(t_{n}\right)\right|=\lambda / 2,\left|y\left(s_{n}\right)\right|=3 \lambda / 4$ and

$$
\begin{gathered}
|y(t)| \geq \varepsilon \text { for } \tau_{n}<t<\sigma_{n}, \\
|y(t)| \leq \varepsilon \text { for } \sigma_{n}<t<\tau_{n+1}, \\
\frac{1}{2} \lambda<|y(t)|<\frac{3}{4} \lambda \text { for } t_{n}<t<s_{n} .
\end{gathered}
$$

By (21), (6), (17) and (12), we have

$$
a f(x(t))=v(t)-c f(y(t)) \geq v_{0}-c f(-\varepsilon)
$$

for $\sigma_{n} \leq t \leq \tau_{n+1}$. For the sake of brevity, let $w_{0}=v_{0}-c f(-\varepsilon)$. Then, $w_{0}$ is positive, because $0<\varepsilon<-f^{-1}\left(-v_{0} / c\right)$. There are two cases to consider: (a) $x(t) \geq f^{-1}\left(w_{0} / a\right)>0$ for $\sigma_{n} \leq t \leq \tau_{n+1}$ and (b) $x(t) \leq f^{-1}\left(-w_{0} / a\right)<0$ for $\sigma_{n} \leq t \leq \tau_{n+1}$. In case (a), using the second equation in system (11) with (5) and (12), we obtain

$$
\begin{aligned}
y^{\prime}(t) & =-a\left(1-e^{-x(t)}\right)-\operatorname{ch}(t)\left(1-e^{-y(t)}\right) \\
& \leq-a\left(1-e^{-x(t)}\right)+c \bar{h} g(y(t)) \\
& \leq-a\left(1-\exp \left(-f^{-1}\left(w_{0} / a\right)\right)\right)+c \bar{h} g(-\varepsilon) \stackrel{\text { def }}{=}-\mu_{1}
\end{aligned}
$$


for $\sigma_{n} \leq t \leq \tau_{n+1}$. From (9), we see that $\mu_{1}$ is positive. Note that $\mu_{1}$ is independent of $n$. Similarly, in case (b), we have

$$
\begin{aligned}
y^{\prime}(t) & =-a\left(1-e^{-x(t)}\right)-c h(t)\left(1-e^{-y(t)}\right) \\
& \geq-a\left(1-e^{-x(t)}\right)-c \bar{h} g(y(t)) \\
& \geq a\left(\exp \left(-f^{-1}\left(-w_{0} / a\right)\right)-1\right)-c \bar{h} g(-\varepsilon) \stackrel{\text { def }}{=} \mu_{2}
\end{aligned}
$$

for $\sigma_{n} \leq t \leq \tau_{n+1}$. From (10), we see that $\mu_{2}$ is also positive and independent of $n$. Let $\mu(\varepsilon)=\min \left\{\mu_{1}, \mu_{2}\right\}>0$. Then, in either case, we get

$$
\left|y^{\prime}(t)\right| \geq \mu \quad \text { for } \sigma_{n} \leq t \leq \tau_{n+1} .
$$

Using this inequality and (8), we can estimate that

$$
\begin{aligned}
2 f^{-1}\left(2 v_{0} / c\right) & \geq\left|y\left(\tau_{n+1}\right)\right|+\left|y\left(\sigma_{n}\right)\right| \geq\left|\int_{\sigma_{n}}^{\tau_{n+1}} y^{\prime}(t) d t\right| \\
& =\int_{\sigma_{n}}^{\tau_{n+1}}\left|y^{\prime}(t)\right| d t \geq \mu\left(\tau_{n+1}-\sigma_{n}\right)
\end{aligned}
$$

or $\tau_{n+1} \leq \sigma_{n}+\Delta$ for $n \in \mathbb{N}$, where $\Delta=2 f^{-1}\left(2 v_{0} / c\right) / \mu$.

Let $I=\bigcup_{n=1}^{\infty}\left[\tau_{n}, \sigma_{n}\right]$. Then, it follows from (11) that $|y(t)| \geq \varepsilon$ for $t \in I$. Hence, by (4), we have

$$
g(y(t)) \geq g(\varepsilon)>0 \quad \text { for } t \in I
$$

and therefore,

$$
\int_{t_{0}}^{\infty} v^{\prime}(t) d t=-c^{2} \int_{t_{0}}^{\infty} h(t) g^{2}(y(t)) d t \leq-c^{2} g^{2}(\varepsilon) \int_{I} h(t) d t
$$

Since

$$
\int_{t_{0}}^{\infty} v^{\prime}(t) d t=\lim _{t \rightarrow \infty} v(t)-v\left(t_{0}\right)=v_{0}-v\left(t_{0}\right)
$$

we obtain

$$
\int_{I} h(t) d t \leq \frac{v\left(t_{0}\right)-v_{0}}{c^{2} g^{2}(\varepsilon)}<\infty .
$$

Hence, from the assumption that $h(t)$ is weakly integrally positive and the estimation that $\tau_{n+1} \leq \sigma_{n}+\Delta$ for $n \in \mathbb{N}$, we see that $\liminf _{n \rightarrow \infty}\left(\sigma_{n}-\tau_{n}\right)=0$. Since $\left[t_{n}, s_{n}\right] \subset\left[\tau_{n}, \sigma_{n}\right]$, it turns out that

$$
\liminf _{n \rightarrow \infty}\left(s_{n}-t_{n}\right)=0 .
$$

By (6) and (7), we have

$$
f(x(t)) \leq \frac{2 v_{0}}{a}
$$

namely,

$$
f^{-1}\left(-2 v_{0} / a\right) \leq x(t) \leq f^{-1}\left(2 v_{0} / a\right)
$$

for $t \geq T_{1}$. From (3), we see that

$$
|x(t)| \leq f^{-1}\left(2 v_{0} / a\right) \quad \text { for } t \geq T_{1} .
$$

Hence, by (5), we obtain

$$
g(x(t)) \leq g\left(-f^{-1}\left(2 v_{0} / a\right)\right) \text { for } t \geq T_{1} .
$$


Using (5) and (13), we have

$$
g(y(t)) \leq g(-3 \lambda / 4) \text { for } t_{n} \leq t \leq s_{n} .
$$

Hence, together with (15), we get

$$
\begin{aligned}
\left|y^{\prime}(t)\right| & \leq a g(x(t))+c \bar{h} g(y(t)) \\
& \leq a g\left(-f^{-1}\left(2 v_{0} / a\right)\right)+c \bar{h} g(-3 \lambda / 4)
\end{aligned}
$$

for $t_{n} \leq t \leq s_{n}$. Letting $\nu=a g\left(-f^{-1}\left(2 v_{0} / a\right)\right)+c \bar{h} g(-3 \lambda / 4)$ and integrating this inequality from $t_{n}$ to $s_{n}$, we obtain

$$
\begin{aligned}
\frac{1}{4} \lambda & =\left|y\left(s_{n}\right)\right|-\left|y\left(t_{n}\right)\right| \leq\left|y\left(s_{n}\right)-y\left(t_{n}\right)\right| \\
& =\left|\int_{t_{n}}^{s_{n}} y^{\prime}(s) d s\right| \leq \int_{t_{n}}^{s_{n}}\left|y^{\prime}(s)\right| d s \leq \nu\left(s_{n}-t_{n}\right) .
\end{aligned}
$$

This contradicts (14). We therefore conclude that $\lim \sup _{t \rightarrow \infty}|y(t)|=\lambda=0$.

In summary, $y(t)$ tends to zero as $t \rightarrow \infty$. Hence, there exists a $T_{3} \geq T_{1}$ such that

$$
|y(t)|<\varepsilon \quad \text { for } t \geq T_{3} .
$$

Using (16) instead of (12) and following the same process as in the above argument, we see that

$$
\left|y^{\prime}(t)\right| \geq \mu \quad \text { for } t \geq T_{3} \text {. }
$$

This inequality yields

$$
\left|y(t)-y\left(T_{3}\right)\right|=\left|\int_{T_{3}}^{t} y^{\prime}(s) d s\right|=\int_{T_{3}}^{t}\left|y^{\prime}(s)\right| d s \geq \mu\left(t-T_{3}\right),
$$

which tends to $\infty$ as $t \rightarrow \infty$. This contradicts the fact that $y(t)$ tends to zero as $t \rightarrow \infty$. Thus, the case of $v_{0}>0$ cannot happen.

The proof of Theorem 1 is now complete.

\section{Discussion}

System (11) is rewritten as a quasi-linear system of the form

$$
\mathbf{x}^{\prime}=A(t) \mathbf{x}+\mathbf{f}(t, \mathbf{x}),
$$

where

and

$$
\mathbf{x}=\left(\begin{array}{l}
x \\
y
\end{array}\right), \quad A(t)=\left(\begin{array}{cc}
0 & c \\
-a & -\operatorname{ch}(t)
\end{array}\right)
$$

$$
\mathbf{f}(t, \mathbf{x})=\mathbf{f}(t, x, y)=\left(\begin{array}{c}
c\left(1-y-e^{-y}\right) \\
-a\left(1-x-e^{-x}\right)-\operatorname{ch}(t)\left(1-y-e^{-y}\right)
\end{array}\right) .
$$

The nonlinear part $\mathbf{f}(t, x, y)$ is a higher-order term with respect to $x$ and $y$, and the linear approximation for system (1) is

$$
\mathbf{x}^{\prime}=A(t) \mathbf{x} .
$$

It is known that under all of the assumptions in Theorem 1, the zero solution of (17) is asymptotically stable, but it is not always uniformly asymptotically stable. For example, if $h(t)=\sin ^{2} t$, then the zero solution of (17) is uniformly asymptotically stable. On the other hand, if $h(t)=1 /(1+t)$ or $h(t)=\sin ^{2} t /(1+t)$, then the 
zero solution of (17) is asymptotically stable, but it is not uniformly asymptotically stable (for details, see [2, 13, 28]).

If the zero solution of a linear system is uniformly asymptotically stable, then the zero solution of the corresponding quasi-linear system is also uniformly asymptotically stable. As Perron [24] has proved, however, the asymptotic stability of the zero solution of a linear system does not necessarily imply that the zero solution of the corresponding quasi-linear system is asymptotically stable. As to Perron's example, see the books [3, pp.42-43], 4, pp. 169-170], [5. p.71], [30, pp.92-93], [32, pp. 315-317], etc.

Therefore, needless to say, even if the zero solution of (17) is asymptotically stable, we cannot show that the zero solution of (1) is globally asymptotically stable. In this paper, we exhibited a sufficient condition for the zero solution of (11) to be globally asymptotically stable as Theorem 1 . This is an advantage of Theorem 1.

The same $h(t)$ put into per capita birth and mortality rates for prey in (E) is a technical setting that makes the modified model still have a unique interior equilibrium point $(c / d, a / b)$. From a biological point of view, however, they should be different. Developing these considerations into a model that provides different time-variational functions on per capita birth and mortality rates of the prey, which is a more biologically practical scenario, will be left for future work.

\section{REFERENCES}

[1] A. Bacciotti and L. Rosier, Liapunov Functions and Stability in Control Theory, 2nd ed., Springer-Verlag, Berlin-Heidelberg-New York, 2005. MR 2146587 (2005m:93001)

[2] R. J. Ballieu and K. Peiffer, Attractivity of the origin for the equation $\ddot{x}+f(t, x, \dot{x})|\dot{x}|^{\alpha} \dot{x}+$ $g(x)=0$, J. Math. Anal. Appl., 65 (1978), 321-332. MR.506309 (80a:34057)

[3] R. Bellman, Stability Theory of Differential Equations, McGraw-Hill, New York-TorontoLondon, 1953; (revised) Dover, Mineola-New York, 2008. MR0061235 (15:794b)

[4] F. Brauer and J. Nohel, The Qualitative Theory of Ordinary Differential Equations, W. A. Benjamin, New York and Amsterdam, 1969; (revised) Dover, New York, 1989.

[5] W. A. Coppel, Stability and Asymptotic Behavior of Differential Equations, Heath, Boston, 1965. MR0190463 (32:7875)

[6] M. J. Crawley (ed.), Natural Enemies: The Population Biology of Predators, Parasites and Diseases, Blackwell Scientific, London-Edinburgh-Boston, 1992.

[7] J. Cronin, Differential Equations: Introduction and Qualitative Theory, 2nd ed., Monographs and Textbooks in Pure and Applied Mathematics, 180, Marcel Dekker, New York-Basel-Hong Kong, 1994. MR:1275827 (95b:34001)

[8] G. F. Gause, The Struggle for Existence, Williams \& Wilkins Co., Baltimore, 1934; (revised) Dover, New York, 2003.

[9] N. J. Gotelli, A Primer of Ecology, 4th ed., Sinauer, Sunderland Associates, 2008.

[10] A. Halanay, Differential Equations: Stability, Oscillations, Time Lags, Academic Press, New York-London, 1966. MR0216103 (35:6938)

[11] J. K. Hale, Ordinary Differential Equations, Wiley-Interscience, New York-London-Sydney, 1969; (revised) Krieger, Malabar, 1980. MR0419901 (54:7918)

[12] L. Hatvani, On the asymptotic stability by nondecrescent Ljapunov function, Nonlinear Anal., 8 (1984), 67-77. MR732416 (85k:34117)

[13] L. Hatvani, On the asymptotic stability for a two-dimensional linear nonautonomous differential system, Nonlinear Anal., 25 (1995), 991-1002. MR.1350721 (96k:34105)

[14] M. P. Hassell, The Dynamics of Arthropod Predator-Prey Systems, Princeton University Press, Princeton, NJ, 1978. MR508052 (80d:92026)

[15] D. W. Jordan and P. Smith, Nonlinear Ordinary Differential Equations: An Introduction to Dynamical Systems, 3rd ed., Oxford Texts in Applied and Engineering Mathematics, 2, Oxford University Press, Oxford, 1999. MR 1743361 (2000j:34001) 
[16] J. P. LaSalle and S. Lefschetz, Stability by Liapunov's Direct Method, with Applications, Mathematics in Science and Engineering, 4, Academic Press, New York-London, 1961. MR0132876 (24:A2712)

[17] A. J. Lotka, Elements of Physical Biology, Williams \& Wilkins Co., Baltimore, 1926.

[18] R. M. May, Stability and Complexity in Model Ecosystems, Princeton University Press, Princeton, NJ, 1973.

[19] D. R. Merkin, Introduction to the Theory of Stability, Texts in Applied Mathematics, 24, Springer-Verlag, New York-Berlin-Heidelberg, 1997. MR1418401 (98f:34074)

[20] A. N. Michel, L. Hou and D. Liu, Stability Dynamical Systems: Continuous, Discontinuous, and Discrete Systems, Birkhäuser, Boston-Basel-Berlin, 2008. MR2351563 (2008i:93001)

[21] L. D. Mueller and A. Joshi, Stability in Model Populations, Princeton University Press, Princeton, NJ, 2000.

[22] W. W. Murdoch and A. Oaten, Predation and population stability, Adv. Ecol. Res., 9 (1975), $1-131$.

[23] A. J. Nicholson and V. A. Bailey, The balance of animal populations, Proc. Zool. Soc. London, 1 (1935), 551-598.

[24] O. Perron, Die Stabilitätsfrage bei Differentialgleichungen, Math. Zeits., 32 (1930), 703-728. MR.1545194

[25] K. P. Persidski, Über die Stabilität einer Bewegung nach der ersten Näherung, Mat. Sb., 40 (1933), 284-293.

[26] N. Rouche, P. Habets and M. Laloy, Stability Theory by Liapunov's Direct Method, Applied Mathematical Sciences, 22, Springer-Verlag, New York-Heidelberg-Berlin, 1977. MR0450715 (56:9008)

[27] J. Sugie, Convergence of solutions of time-varying linear systems with integrable forcing term, Bull. Austral. Math. Soc., 78 (2008), 445-462. MR2472280 (2009k:34102)

[28] J. Sugie, Influence of anti-diagonals on the asymptotic stability for linear differential systems, Monatsh. Math., 157 (2009), 163-176. MR2504784 (2010d:34103)

[29] J. Sugie and Y. Ogami, Asymptotic stability for three-dimensional linear differential systems with time-varying coefficients, Quart. Appl. Math., 67 (2009), 687-705. MR2588230 (2010k:34141)

[30] F. Verhulst, Nonlinear Differential Equations and Dynamical Systems, Springer-Verlag, New York-Berlin-Heidelberg, 1990. MR.1036522 (91b:34002)

[31] V. Volterra, Leçons sur la Théorie Mathématique de la Lutte pour la Vie, GauthierVillars, Paris, 1931. Reprinted in Les Grands Classiques Gauthier-Villars, 1990. MR 1189803 (93k:92011)

[32] H. K. Wilson, Ordinary Differential Equations, Introductory and Intermediate Courses Using Matrix Methods, Addison-Wesley, Massachusetts-California-London-Ontario, 1971. MR0280764 (43:6483)

[33] T. Yoshizawa, Stability Theory by Liapunov's Second Method, Math. Soc. Japan, Tokyo, 1966. MR0208086 (34:7896)

[34] T. Yoshizawa, Stability Theory and the Existence of Periodic Solutions and Almost Periodic Solutions, Applied Mathematical Sciences, 14, Springer-Verlag, New York-Heidelberg-Berlin, 1975. MR0466797 (57:6673)

Department of Mathematics and Computer Science, Shimane University, Matsue 6908504, JAPAN

E-mail address: jsugie@riko.shimane-u.ac.jp

Department of Mathematics, Pusan National University, Busan 609-735, Republic of Korea

Current address: Department of Mathematics, Chonnam National University, Gwangju 500757, Republic of Korea

E-mail address: saito.yasuhisa@gmail.com

School of Mathematics and Statistics, Northeast Normal University, Changchun, 130024 Jilin, People's Republic of China

E-mail address: mfan@nenu.edu.cn 